\title{
Pd nanoparticles immobilized on halloysite decorated with a cyclodextrin modified melamine-based polymer: a promising heterogeneous catalyst for hydrogenation of nitroarenes
}

Samahe Sadjadi ${ }^{1}$, Maryam Akbari2, Eric Monflier ${ }^{3}, \underline{\text { Majid M. Heravi }}{ }^{2}$, Bastien Leger $^{3}$

1- Gas Conversion Department, Faculty of Petrochemicals, Iran Polymer and Petrochemicals Institute, PO Box 14975-112, Tehran, Iran

2- Department of Chemistry, School of Science, Alzahra University, PO Box 1993891176, Vanak, Tehran, Iran

3- Univ. Artois, CNRS, Centrale Lille, ENSCL, Univ. Lille, UMR 8181, Unité de Catalyse et de Chimie du Solide (UCCS), Lens, F-62300, France

\begin{abstract}
For the first time, a hybrid system composed of halloysite (Hal) and a cyclodextrin modified melamine-based polymer is developed and employed for immobilization of $\operatorname{Pd}(0)$ nanoparticles. The resulting catalytic hybrid system, Pd@HTMC, was then applied as a heterogeneous catalyst for hydrogenation of nitroarenes to the corresponding anilines. The results established that Pd@HTMC could promote hydrogenation under very mild reaction conditions and in a relatively short time. Moreover, investigation of a sterically demanding substrate, nitro-naphthalene, as well as a substrate with a competing functional group, 4-nitroacetophenone, confirmed that the catalyst is capable of hydrogenation of a sterically demanding substrate while selectively hydrogenating the nitro functional group over keto-functionality. The
\end{abstract}


Source: Sadjadi S, Akbari M, Monflier E, Heravi MM, Leger B. Pd nanoparticles immobilized on halloysite decorated with a cyclodextrin modified melamine-based polymer: a promising heterogeneous catalyst for hydrogenation of nitroarenes. New J Chem. 2018;42(19):15733-42. DOI: 10.1039/C8NJ03014F

recyclability experiment proved that the catalyst was recyclable up to six reaction runs with a slight loss of the catalytic activity and Pd leaching. Moreover, a hot filtration test confirmed the heterogeneous nature of the catalyst. Finally, the contribution of the cyclodextrin modified melamine-based polymer to the catalysis was confirmed by comparison of the catalytic activity of Pd@HTMC with control catalysts. 